\title{
Polarization-dependent circular Dammann grating made of azo-dye-doped liquid crystals
}

\author{
Dan Luo, ${ }^{1}$ Xiao Wei Sun, ${ }^{1,2, \star}$ Hai Tao Dai, ${ }^{2}$ and Hilmi Volkan Demir ${ }^{1,3,4}$ \\ ${ }^{1}$ School of Electrical and Electronic Engineering, Nanyang Technological University, \\ Nanyang Avenue, Singapore 639798, Singapore \\ 2Department of Applied Physics, College of Science, Tianjin University, Tianjin 300072, China \\ ${ }^{3}$ School of Physical and Mathematical Sciences, Nanyang Technological University, \\ Nanyang Avenue, Singapore 639798, Singapore \\ ${ }^{4}$ Department of Electrical and Electronics Engineering and Department of Physics, \\ Bilkent University, Bilkent, Ankara, 06800, Turkey \\ *Corresponding author: exwsun@ntu.edu.sg
}

Received 28 October 2010; revised 2 March 2011; accepted 30 March 2011; posted 31 March 2011 (Doc. ID 137203); published 19 May 2011

\begin{abstract}
A polarization-dependent circular Dammann grating (CDG) was generated from an azo-dye-doped liquid crystal (LC) cell. A simple multiexposure photo-alignment process was used to fabricate a binary phase LC CDG zone plane, which was composed of an odd zone with a twisted nematic LC structure and an even zone with a homogenous LC structure. A two-order CDG with equal-intensity rings was produced through a Fourier transform. The maximum zeroth and first diffraction orders of obtained CDG can be separately achieved by rotating the analyzer's polarization direction. The CDG using an azodye-doped LC cell can be used to generate diffractions by lasers in a broad wavelength range, hence expanding possible device applications. (C) 2011 Optical Society of America
\end{abstract}

OCIS codes: $\quad 230.3720,050.1970,110.5220$.

\section{Introduction}

A circular Dammann grating (CDG) is a diffraction grating that generates a circular equal-intensity pattern at the image plane [1]. Circular Dammann gratings with periodic structures have been designed using the Hankel transform method $[2,3]$ and the circular spot rotation method [4]. The phase modulation of the CDG can be binary [2-4], which is the simplest type, multilevel [5], or continuous. As a useful diffractive optical element, the CDG was reported to be a useful measurement tool for quick determination of the back focal length of a lens [6]. The separation of rings in the focal length of a $C \bar{D} G$ can be used in collimation testing of a laser beam [7]. Wen et al. reported the practical application of a CDG in

0003-6935/11/152316-06\$15.00/0

(C) 2011 Optical Society of America angular and area measurement, where angles and areas can be rapidly obtained with maintained accuracy [ 8,9$]$. In addition, the CDG was also used in an optical image coding system [10].

Azo dye is a kind of photosensitive material that is capable of aligning liquid crystal (LC) molecules through a photoisomerization molecular reorientation process $[11,12]$. Azo-dye-doped nematic LC is a good candidate for fabricating polarization-related optical devices, such as polarization Fresnel lenses $[13,14]$, polarization gratings [15-17], vertical photonic crystals [18], polarization rotators [19], and photonic crystal fiber devices [20].

Previously reported CDGs were usually fabricated by optical-inactive materials (resist, glass etc.), suitable only for a single wavelength due to the requirement of wavelength-dependent $\pi$ phase difference for high diffractive efficiency. In consequence, the application of such CDGs is limited. In this paper, 
a broad band polarization-dependent CDG was generated from an azo-dye-doped nematic LC cell. A binary phase LC two-order CDG zone plane consisting of an odd zone with a twisted nematic (TN) LC structure and an even zone with a homogenous LC structure was fabricated through a multiexposure photoalignment process. The maximum zeroth and first/second diffraction orders of a CDG can be separately achieved by changing the analyzer's direction, thus leading to a polarization-dependent tunable CDG. The CDG based on azo-dye-doped LC can be operated in a broad wavelength range due to the waveguide property of the TN LC structure.

A binary phase CDG, as a kind of radially periodic phase grating, can generate impulse rings with equal intensity in the far field. The number of the diffractive order with equal intensity in the spectral domain is defined as the order of a CDG. A radially periodic image $g(r)$ with period $T$ can be decomposed into a linear combination of circular sinusoid functions as [2]

$$
g(r)=\sum_{n=1}^{\infty} c_{n} \sin \left(2 \pi \frac{n}{T} r\right)
$$

where the $n$th term represents the $n$th ring. Its Fourier spectrum calculated from Fourier transform (or Hankel transform) is given by [2]

$$
G(q)=\frac{1}{\sqrt{\pi}} \sum_{n=1}^{\infty} c_{n} \frac{n / T}{(n / T+q)^{3 / 2}} \delta^{(1 / 2)}(q-n / T),
$$

where $q=\rho / \lambda f$ is the spatial frequency, with $\rho$ representing a polar coordinate in the observed plane and $f$ representing the focal length of the lens, and $\delta^{(1 / 2)}(x)$ is the half-order derivative of the impulse $\delta(x)$. For the case of a two-order CDG with a profile as shown in Fig. $1(\mathrm{a})$, where $\left\{r_{1}=0.1634, r_{2}=0.5, r_{3}=0.8366\right.$, $r_{4}=\overline{1\}}$ are the normalized phase transitions in a period length and are chosen from [2] directly, the corresponding theoretical spectral intensity in the far field is presented in Fig. 1(b), and the theory diffraction efficiency limit is $65.2 \%$. The calculated coefficients

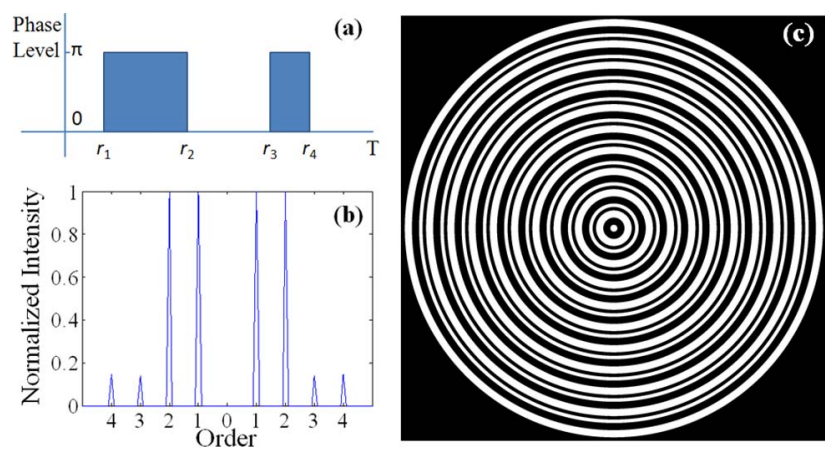

Fig. 1. (Color online) (a) Cross section of a two-order binary phase $(0, \pi)$ CDG, (b) theoretical normalized intensity of a two-order CDG, (c) two-order binary phase CDG pattern transferred onto a photomask. $c_{n}$ are given by

$$
c_{n}=\frac{2}{n \pi}\left[-2 \cos \left(2 \pi n x_{1}\right)+(-1)^{n+2}+1\right],
$$

where $x_{1}=r_{1}=0.1634$. The designed two-order binary phase CDG pattern was then transferred to a photomask with a period of $T=200 \mu \mathrm{m}$ and a total aperture of $8 \mathrm{~mm}$, as depicted in Fig. 1(c).

\section{Experiment and Simulation}

The materials used herein consisted of $99 \mathrm{wt} . \% \mathrm{LC}$, E7 $\left(n_{o}=1.5216\right.$ and $\left.n_{e}=1.7462\right)$ from Merck, and 1 wt. \% azo dye, Methyl Red (MR) from Aldrich. Two indium tin oxide (ITO) coated glass slides were used to assemble an empty cell with a gap of $d=30 \mu \mathrm{m}$. The dye-doped LC mixture was then infiltrated into an empty cell. As both glass substrates were not rubbed, the alignment of LC in the cell was initially random.

The LC molecules in the cell can be reoriented by linearly polarized light in the absorption band of azo dye. When excited by light in the absorption spectrum, the azo dye in the LC sample would undergo trans-cis isomerization, followed by molecular reorientation, diffusion, and adsorption on the ITO glass surface. The adsorbed dyes then align liquid crystals by forcing the LC molecules to reorient along the direction of the azo dye's long axis $[13,14]$.

In our experiment, a diode-pumped linearly polarized solid state laser light at a wavelength of $532 \mathrm{~nm}$, which is in the absorption band of MR dye $(410-550 \mathrm{~nm}$, with its peak around $500 \mathrm{~nm})$, was used to irradiate the dye-doped LC sample for photoaligning LC molecules in the cell. The absorption of MR dye at $532 \mathrm{~nm}$ is high and is about $70 \%$ of that at peak absorption wavelength of $500 \mathrm{~nm}$. The photoalignment process for fabricating a CDG on the azodye-doped LC sample consists of two steps. In the first step, as shown in Figs. 2(a)-2(c), glass substrates 1 and 2 were illuminated by the laser light with an intensity level of $120 \mathrm{~mW} / \mathrm{cm}^{2}$ for $3 \mathrm{~min}$ with linear polarization along the $x$ axis separately. Then the MR dye on the substrate aligned the LC to be perpendicular to the incident light polarization, leading to a parallel alignment along the $y$ axis of the LC on both substrates and, therefore, to the formation of a homogeneous LC layer structure. In the second step, glass substrate 2 , covered by the photomask with a CDG pattern, was illuminated by laser with linear polarization along the $y$ axis. The odd circle/rings of the CDG are transparent, and the even rings are opaque, as shown in Fig. 2(d). During the second illumination, the MR dye on substrate 2 reoriented the LC again, and a TN structure for the odd zone was finally achieved, while the homogenous structure of the even zone remained unchanged, as shown in Fig. 2(e). The adsorption-induced twist angle, defined as the angle between the original LC director's direction and the reoriented LC molecule director's by azo dye, is dependent on the exposure time 
and pumping intensity of illumination light [19]. In our experiment, for demonstrating a more general case, a $60^{\circ} \mathrm{TN}$ structure for the odd circle/rings of the CDG zone was obtained using a laser intensity of $120 \mathrm{~mW} / \mathrm{cm}^{2}$ and exposure time of $2 \mathrm{~min}$ for the second illumination in Fig. 2(d). A larger twist angle TN structure (e.g., $90^{\circ} \mathrm{TN}$ ) could be obtained by lengthening the exposure time or increasing the illumination intensity. Such a multiexposure photoalignment process provides a simple way to align the LC in the cell, as no rubbing is needed.

For the odd zone of the cell with $\theta^{\circ} \mathrm{TN}$ structure (where $\theta$ is the twist angle between the LC directors on substrate 1 and 2) as presented in Fig. 2(e), if light with linear polarization along the $y$ axis (parallel to the LC director on substrate 1) is incident from the substrate 1 side, the $\theta^{\circ} \mathrm{TN}$ structure will rotate the polarization of light by $\sim \theta^{\circ}$ after it passes through the cell when Mauguin's condition is satisfied $\left(\Delta n d \gg \lambda\right.$, where $\Delta n=n_{e}-n_{o}$ and $\lambda$ is wavelength of incident light) [21], which means the polarization of output light passing through the odd zone will be rotated to be parallel to the LC director on substrate 2 . In our experiment, Mauguin's condition was satisfied and $\theta$ was equal to $60^{\circ}$. For the even zone with homogeneous structure, the polarization of incident light would not be changed after it passes through the cell, when light with polarization along the $y$ axis is incident from substrate 1 .

When the LC cell sample was examined under an optical microscope with two polarizers, the white light source would pass through polarizer 1 (P1), substrate 1, LC cell, substrate 2 , and polarizer $2(\mathrm{P} 2)$ in sequence in our experiment. The direction of polarizer 1 was set to be parallel to the LC director on substrate 1 , both along the $y$ axis. The obtained microscope images of the photoaligned azo-dye-doped LC with a CDG pattern are shown in Fig. 3, where Figs. $3(\mathrm{a})$ and $3(\mathrm{~b})$ represent images captured with

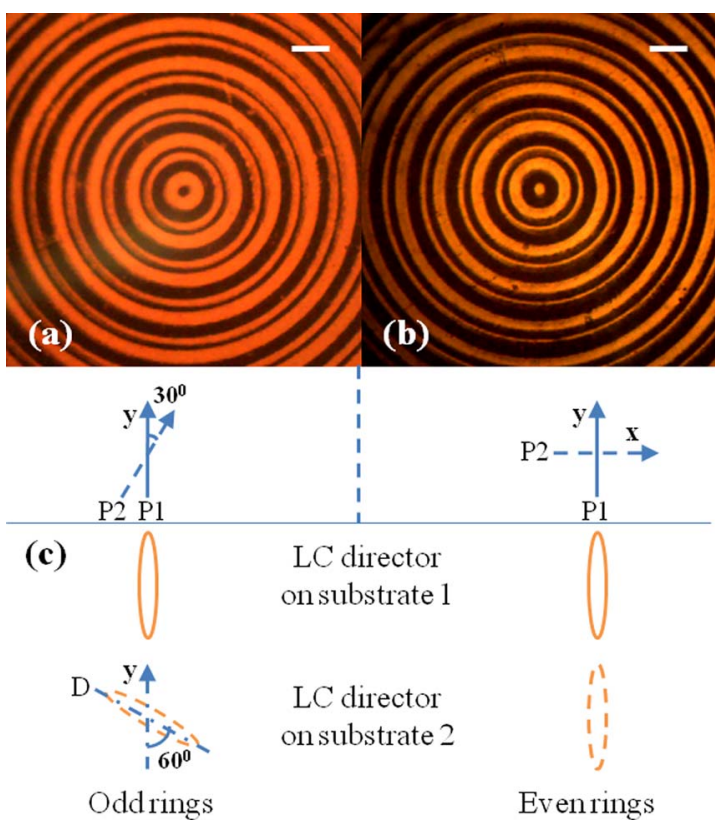

Fig. 3. (Color online) Optical microscope images of the photoaligned azo-dye-doped LC cell with CDG pattern in the cases of (a) $30^{\circ}$ angle of polarizer-polarizer and (b) crossed polarizerpolarizer. Solid line and dashed line represent polarizer 1 and polarizer 2 , respectively. The scale bars are $200 \mu \mathrm{m}$. (c) The directions of LC directors on substrates 1 and 2 are shown for odd and even ring zones, respectively.

polarizer-polarizer angles of $30^{\circ}$ and $90^{\circ}$, corresponding to a completely dark odd zone and a completely dark even zone respectively. The directions of polarizer 1 and polarizer 2 for each case are drawn below each image. For the odd zone, a completely darkened image appeared only when polarizer 2 had a $30^{\circ}$ angle with polarizer 1 as shown in Fig. 3(a), indicating that the LC director (D) on substrate 2 should be perpendicular to polarizer 2 and has a $60^{\circ}$ angle with the $y$ axis, as shown in

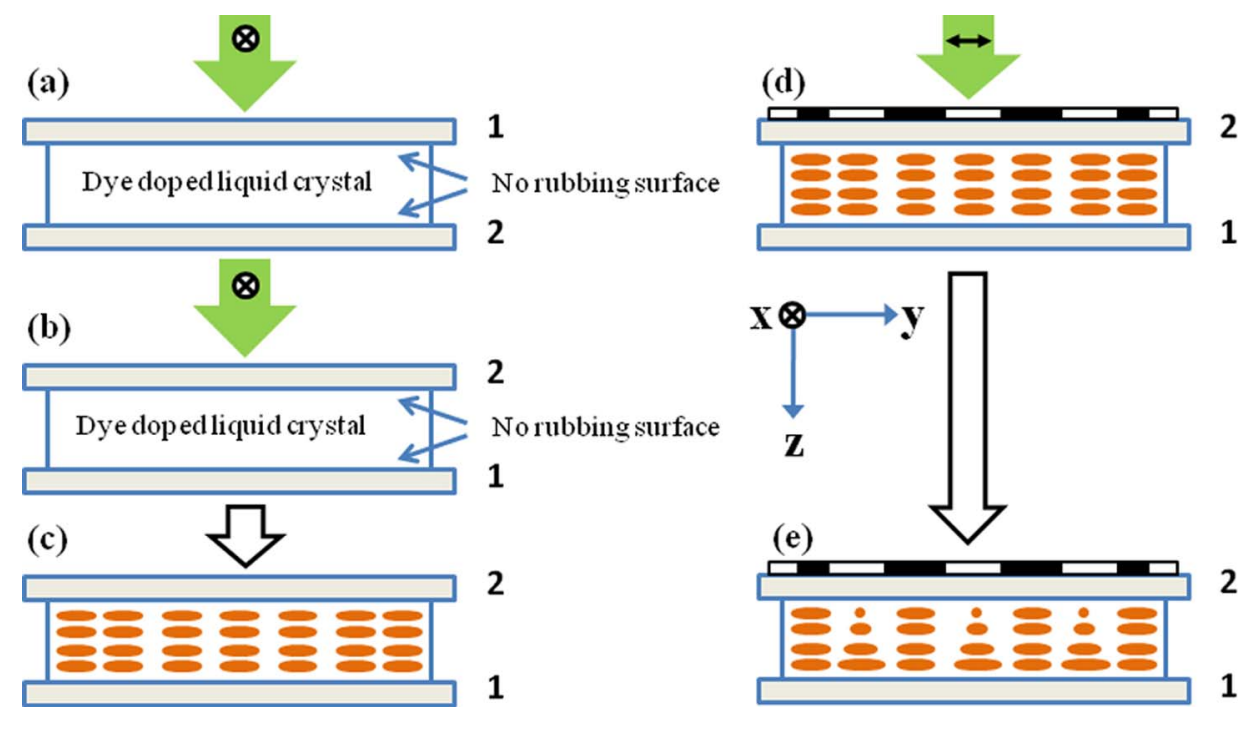

Fig. 2. (Color online) Schematic fabrication process of azo-dye-doped LC CDG. 
the left part of Fig. 3(c). Therefore, a $60^{\circ} \mathrm{TN}$ structure can be confirmed in the odd circle/ring zone. Similarly, for the even ring zone, a completely darkened image appeared only when polarizer 2 was perpendicular to polarizer 1, as shown in Fig. 3(b), confirming a parallel alignment LC structure.

For generating CDG diffraction, the azo-dye-doped LC cell sample recorded with a CDG pattern was illuminated by a collimated He-Ne laser $(633 \mathrm{~nm})$ with a beam diameter of about $1 \mathrm{~cm}$ and intensity of $0.2 \mathrm{~mW} / \mathrm{cm}^{2}$ to generate the CDG. The absorption of MR at $633 \mathrm{~nm}$ is very low and is around $10 \%$ of that at peak absorption wavelength of $500 \mathrm{~nm}$. A polarizer was used to ensure that the polarization of incident light was parallel to the LC director on substrate 1 (both along the $y$ axis). A spherical lens $(f=20 \mathrm{~cm})$ placed right behind the LC cell sample was used to perform the Fourier transform of the CDG pattern. An analyzer was used to verify the image polarization. The circular diffraction pattern was obtained at the focal plane of the Fourier-transform lens and then captured by a CCD camera.

\section{Results and Discussions}

Figure $\underline{4}$ shows the polarization-dependent property of the azo-dye-doped LC CDG recorded by CCD. The polarization of the incident light, parallel to the LC directors on substrate 1 in the even zone, would not change, because the even zone has a homogeneous LC structure, while the polarization of the incident light through the odd zone is rotated by $\sim 60^{\circ}$, because the odd zone has $60^{\circ} \mathrm{TN} \mathrm{LC}$ structure. That is called the waveguiding effect [21] in the TN LC structure. As a result, the angle between the polarizations of output light from the even and odd zones is around $60^{\circ}$. Figure 4 presents the observed images recorded by a CCD with (a) no analyzer, (b) $30^{\circ}$, (c) $15^{\circ}$, (d) $0^{\circ}$, (e) $-15^{\circ}$, (f) $-30^{\circ},(\mathrm{g})-45^{\circ}$, and (h) $-60^{\circ}$ of analyzerpolarizer angle, where $0^{\circ}$ is along the $y$ axis. Corresponding output light polarization from the even $\left(E_{\text {even }}\right)$ and odd $\left(E_{\text {odd }}\right)$ zones, polarization $(P)$, and analyzer $(A)$ directions are indicated below each image, respectively.

Figure 4(a) shows the observed image obtained without using an analyzer. We can see that both the zeroth and first/second diffraction orders corresponding to the light spot and CDG two equalintensity rings, respectively, existed in this case. Figures 4(b) and 4(h) show the observed images with the maximum zeroth and first/second diffraction orders, where the analyzer was set to $30^{\circ}$ and $-60^{\circ}$, respectively, compared to the polarizer direction $(y$ axis). The reason behind this observed image can be found as follows. Assuming the incident light
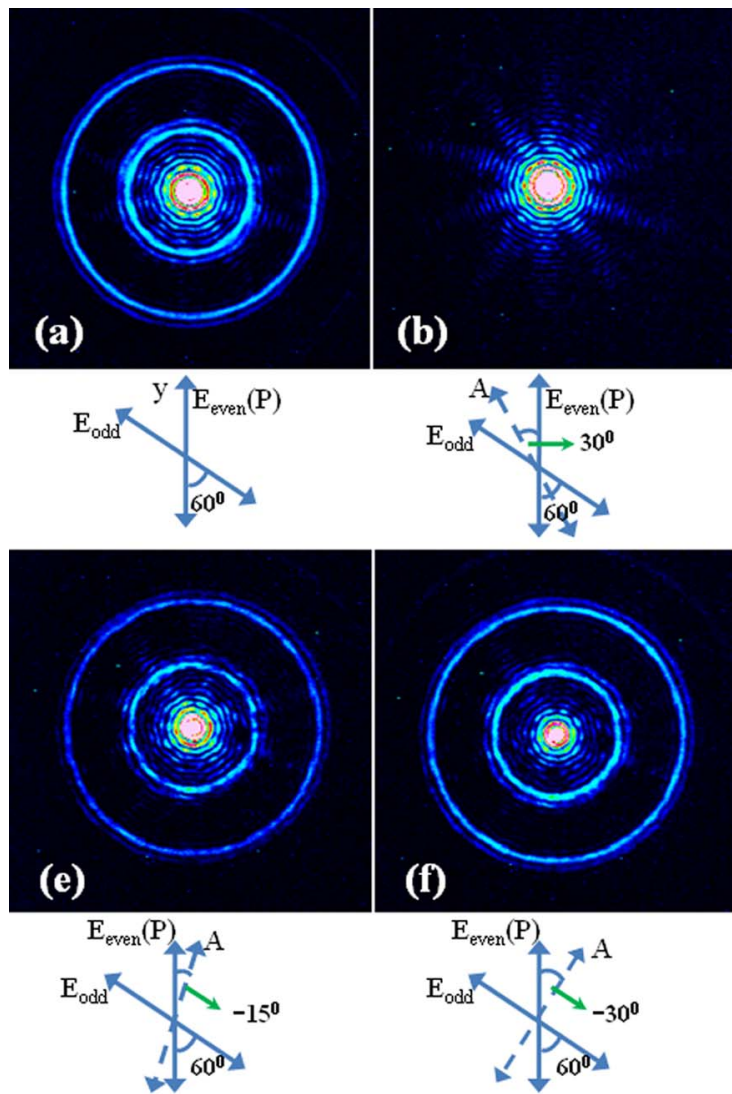
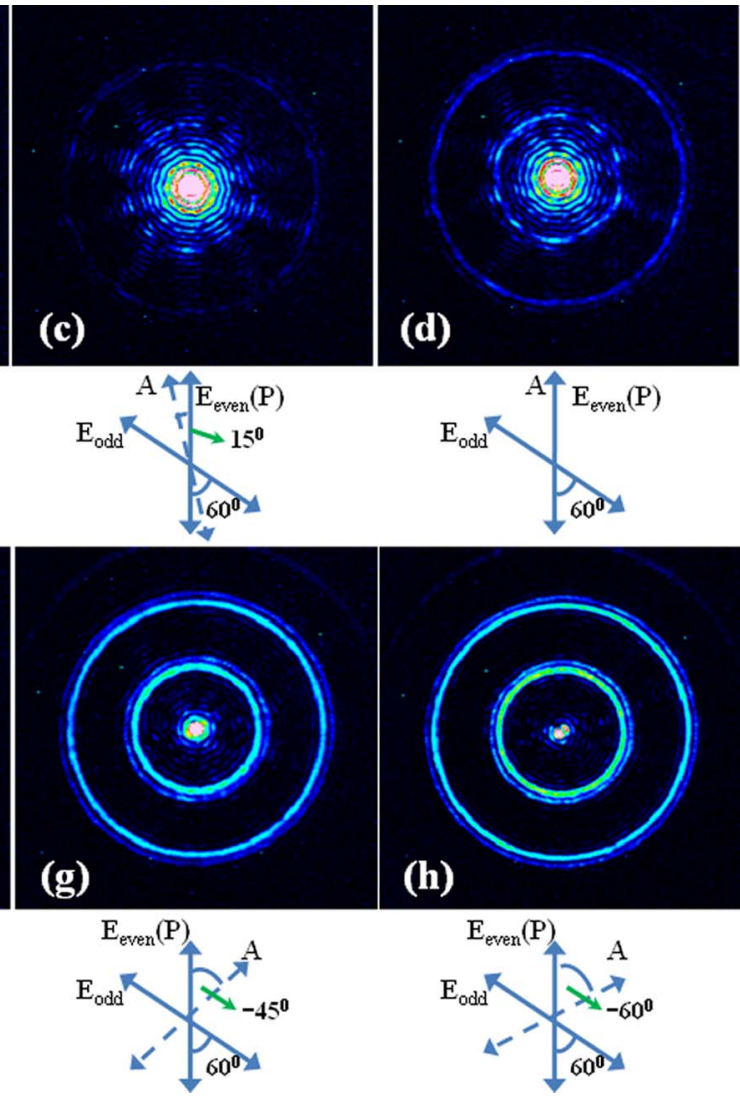
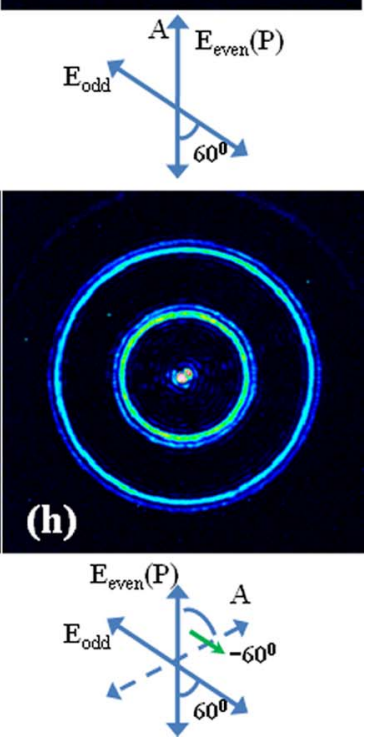

Fig. 4. (Color online) Observed images recorded by a CCD (a) without an analyzer and with (b) $30^{\circ}$, (c) $15^{\circ}$, (d) $0^{\circ}$, (e) $-15^{\circ}$, (f) $-30^{\circ}$, (g) $-45^{\circ}$, and $(\mathrm{h})-60^{\circ}$ analyzer-polarizer angle, where $0^{\circ}$ is along the $y$ axis. Corresponding output light polarization from even $\left(E_{\text {even }}\right)$ and odd $\left(E_{\text {odd }}\right)$ zones, polarization $(P)$, and analyzer $(A)$ directions are shown below each image. 
has a polarization state parallel to $0^{\circ}$, after passing through the CDG, the polarization of output light in the even zone remained unchanged, while that in the odd zone became parallel to $60^{\circ}$, defined in Fig. 4 . When the analyzer was set along the direction of $30_{\rightarrow}^{\circ}$ defined in Fig. 4, the electric field components of $\boldsymbol{E}_{\text {even }}$ and $\boldsymbol{E}_{\text {odd }}$ along the direction of the analyzer are equal to each other, with both the same amplitude and phase, leading to a zero phase difference of output light from the even and odd zones and finally the zeroth diffraction order maximum. In contrast, when the analyzer was set along $-60_{\rightarrow}^{\circ}$ defined in Fig. 4, the electric field components of $\boldsymbol{E}_{\text {even }}$ and $\boldsymbol{E}_{\text {odd }}$ along the direction of analyzer have the same amplitude but a $\pi$ phase difference, leading to a $\pi$ phase difference of output light from the even and odd zones and finally the desired CDG image with the first/second diffraction order maximum. If the analyzer was set between $30^{\circ}$ and $-60^{\circ}$, the obtained images would be in one of the intermediate states with both zeroth and first/second orders, and these corresponding images are given in Figs. 4(c) $-4(\mathrm{~g})$. Therefore, by adjusting the analyzer's direction, we can gradually change the output CDG image, which makes it possible to use it as a polarizationdependent tunable optical element.

From Fig. 4(h), we can see that two equal-intensity rings are produced from our sample as predicted; the unwanted zero order in the CDG image was due to its high sensitivity to fabrication errors, where a very bright zero order can be produced even when a small phase error exists [2]. However, this did not prevent us from demonstrating the utility of the polarizationdependent azo-dye-doped LC CDG. The splitting of the main intensity maximum is due to the property of the binary phase CDG and could be eliminated by using a four-phase-level CDG [2].

Figure 5 shows the normalized intensity of the two-order binary CDG given in Fig. 4(h), where $\rho$ represents the distance from the center of CDG in the polar coordinates. The splitting of the main intensity maximum, as discussed previously by S. Zhao et al. $[2,6]$, is due to the property of binary phase CDG and could be eliminated by a four-phase-level CDG [2]. The radii of produced intensity rings can be calculated from $q=\rho / \lambda f=n / T$, and the theoretical radius of the first and second order rings on the image plane are $\rho_{1}=\lambda f / T=633 \mu \mathrm{m}$ and $\rho_{2}=2 \lambda f / T=$ $1266 \mu \mathrm{m}$, respectively, where $\lambda=633 \mathrm{~nm}, f=20 \mathrm{~cm}$, and $T=200 \mu \mathrm{m}$ in our experiment. The obtained experimental $\rho_{1}$ and $\rho_{2}$ were around $606 \mu \mathrm{m}$ and $1263 \mu \mathrm{m}$, respectively, slightly smaller than what is predicted, which was due to the errors of the radii's positions in the binary phase CDG [2]. Therefore, our experimental results are quite consistent with the simulation.

It is worth noting that any laser 1) satisfying Mauguin's condition and 2) that is sufficiently far away from MR azo-dye absorption peak, or even near the absorption peak but then with low intensity, could be used to generate the polarization-dependent

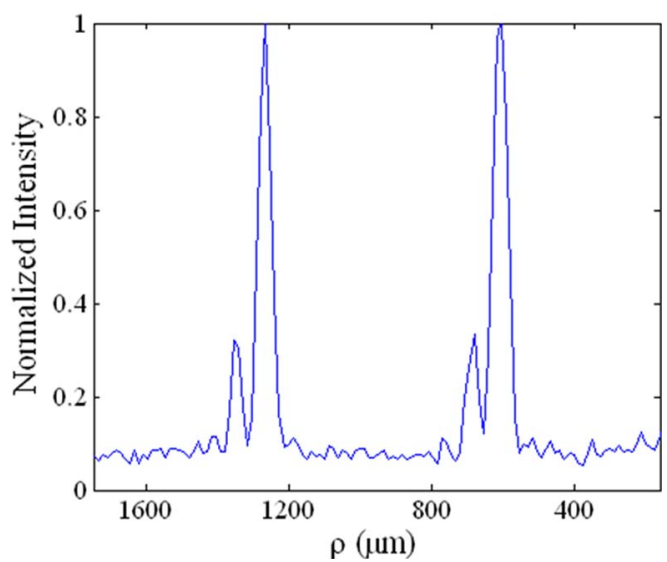

Fig. 5. (Color online) Cross section of normalized intensity of the two-order binary CDG obtained in the experiment.

CDG diffraction due to the light-rotating (waveguiding) property of TN structure in the odd zone. This broadband property expands the CDG application range, which is of great advantage compared to previously reported CDGs operating at only one fixed working wavelength.

\section{Conclusion}

In conclusion, we have generated a polarizationdependent CDG diffraction from an azo-dye-doped LC cell. A simple multiexposure photoaligned process was used to fabricate a binary phase LC CDG zone plane that consisted of an even zone with a homogenous LC structure and an odd zone with a $60^{\circ} \mathrm{TN}$ LC structure. The maximum zeroth and first/ second diffraction orders of the CDG can be separately achieved by changing the analyzer's polarization direction. If the chosen analyzer's direction leads to a zero phase difference in output light from the even and odd zones, the maximum zeroth diffraction order will be achieved. In contrast, if the chosen analyzer's direction leads to a $\pi$ phase difference in output light from the even and odd zones, the maximum first diffraction order will be produced. The CDG diffraction from our azo-dye-doped LC cell can be generated by lasers within a broad wavelength band, which expands possible applications.

\section{References}

1. C. Zhou, J. Jia, and L. Liu, "Circular Dammann grating," Opt. Lett. 28, 2174-2176 (2003).

2. S. Zhao and P. S. Chung, "Design of a circular Dammann grating," Opt. Lett. 31, 2387-2389 (2006).

3. F. J. Wen and P. S. Chung, "A new circular Dammann grating using a Hankel transform," J. Opt. A: Pure Appl. Opt. 10, 075306 (2008).

4. F. J. Wen, S. Y. Law, and P. S. Chung, "Design of circular Dammann gratings by employing the circular spot rotation method," Appl. Opt. 46, 5452-5455 (2007).

5. U. Levy, B. Desiatov, I. Goykhman, T. Nachmias, A. Ohayon, and S. E. Meltzer, "Design, fabrication, and characterization of circular Dammann gratings based on grayscale lithography," Opt. Lett. 35, 880-882 (2010). 
6. S. Zhao, J. F. Wen, and P. S. Chung, "Simple focal-length measurement technique with a circular Dammann grating," Appl. Opt. 46, 44-49 (2007).

7. S. Zhao and P. S. Chung, "Collimation testing using a circular Dammann grating," Opt. Commun. 279, 1-6 (2007).

8. F. J. Wen and P. S. Chung, "Use of the circular Dammann grating in angle measurement," Appl. Opt. 47, 5197-5200 (2008).

9. F. J. Wen, Z. Chen, and P. S. Chung, "Area measurement at long-distance using a circular Dammann grating," Appl. Opt. 49, 648-652 (2010).

10. K. B. Doh, K. Dobson, T.-C. Poon, and P. S. Chung, "Optical image coding with a circular Dammann grating," Appl. Opt. 48, 134-139 (2009).

11. K. Ichimura, "Photoalignment of liquid-crystal systems," Chem. Rev. 100, 1847-1874 (2000).

12. A. G. Chen and D. J. Brady, "Real-time holography in azo-dyedoped liquid crystals," Opt. Lett. 17, 441-443 (1992).

13. T.-H. Lin, Y. H. Huang, A. Y. G. Fuh, and S.-T. Wu, "Polarization controllable Fresnel lens using dye-doped liquid crystals," Opt. Express 14, 2359-2364 (2006).

14. L.-C. Lin, H.-C. Jau, T.-H. Lin, and A. Y.-G. Fuh, "Highly efficient and polarization independent Fresnel lens based on dye-doped liquid crystal," Opt. Express 15, 2900-2906 (2007).

15. J.-R. Wang, C.-R. Lee, and M.-R. Lee, "Photorefractive effect induced by polarization gratings in dye-doped liquid crystals," Opt. Lett. 29, 110-112 (2004).

16. S. P. Gorkhali, S. G. Cloutier, and G. P. Crawford, "Stable polarization gratings recorded in azo-dye-doped liquid crystals," Appl. Phys. Lett. 88, 251113 (2006).

17. T. Sasaki, H. Ono, N. Kawatsuki, and M. Kuwabara, "Liquid crystal phase gratings using photoregulated photocrosslinkable polymer liquid crystals," Appl. Phys. Lett. 87, 161112 (2005).

18. S. P. Gorkhali, S. G. Cloutier, and G. P. Crawford, "Twodimensional vectorial photonic crystals formed in azo-dyedoped liquid crystals," Opt. Lett. 31, 3336-3338 (2006).

19. C.-Y. Huang, H.-Y. Tsai, Y.-H. Wang, C.-M. Huang, K.-Y. Lo, and C.-R. Lee, "Linear polarization rotators based on dyedoped liquid crystal cells," Appl. Phys. Lett. 96, 191103 (2010).

20. T. T. Alkeskjold, J. Lægasgaard, and A. Bjarklev, "All-optical modulation in dye-doped nematic liquid crystal photonic bandgap fibers," Opt. Express 12, 5857-5871 (2004).

21. P. Yeh and C. Gu, "Jones matrix method," in Optics of Liquid Crystal Displays, 2nd ed. (Wiley, 2010), pp. 173-289. 